\title{
Unraveling the Hook Effect: A Comprehensive Study of High Antigen Concentration Effects in Sandwich Lateral Flow Immunoassays
}

\author{
Georgina M. S. Ross, Daniel Filippini, Michel W. F. Nielen, and Gert IJ. Salentijn*
}

Cite This: Anal. Chem. 2020, 92, 15587-15595

Read Online

ACCESS

Llll Metrics \& More

回国 Article Recommendations

Supporting Information

ABSTRACT: Sandwich lateral flow immunoassays (LFIAs) are limited at high antigen concentrations by the hook effect, leading to a contradictory decrease in the test line $(T)$ intensity and falsenegative results. The hook effect is mainly associated with the loss of $\mathbf{T}$, and research focuses on minimizing this effect. Nevertheless, the control line (C) intensity is also affected at higher analyte concentrations, undesirably influencing the $\mathrm{T} / \mathrm{C}$ ratio in LFIA readers. The main aim of this work is to identify and understand these high antigen concentration effects in order to develop ubiquitous strategies to interpret and mitigate such effects. Four complementary experiments were performed: performance assessment of three different allergen LFIAs (two for hazelnut, one for

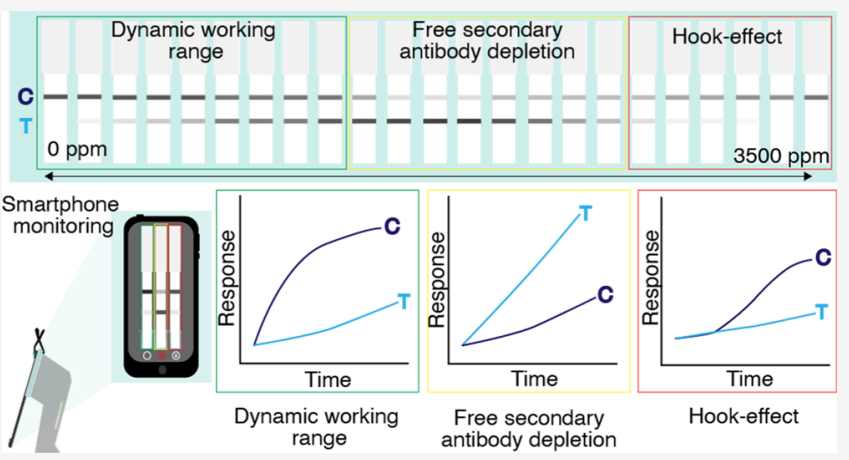
peanut) over $0.075-3500 \mathrm{ppm}$, LFIAs with C only, surface plasmon resonance (SPR) binding experiments on the immobilized control antibody, and smartphone video recording of LFIAs during their development. As antigen concentrations increase, the $\mathbf{C}$ signal decreases before the $\mathbf{T}$ signal does, suggesting that distinct mechanisms underlie these intensity reductions. Reduced binding at the $\mathbf{C}$ occurred even in the absence of $\mathbf{T}$, so the upfront $\mathbf{T}$ does not explain the loss of $\mathbf{C}$. SPR confirmed that the $\mathbf{C}$ antibody favors binding with free labeled antibody compared with a labeled antibody-analyte complex, indicating that in antigen excess, binding is reduced at $\mathbf{C}$ before $\mathbf{T}$. Finally, a smartphone-based video method was developed for dynamically monitoring the LFIA development in real time to distinguish between different concentration-dependent effects. Digitally analyzing the data allows clear differentiation of highly positive samples and false-negative samples and can indicate whether the LFIA is in the dynamic working range or at critically high concentrations. The aim of this work is to identify and understand such high antigen concentration effects in order to develop ubiquitous strategies to interpret and mitigate such effects.

$\mathrm{L}$ ateral flow immunoassays (LFIAs) have revolutionized consumer diagnostics, translating laboratory-based immunoassays into affordable and accessible home testing devices. ${ }^{1}$ Sandwich-format LFIAs utilize two bivalent monoclonal antibodies (mAbs) to capture and detect large multivalent targets, such as allergens. ${ }^{2}$ In microplate-based immunoassays, capture $\mathrm{mAbs}$ are directly immobilized onto the solid support of the microwell; ${ }^{3}$ in LFIA, the capture $\mathrm{mAb}$ is shaped into a test line $(T)$. A labeled secondary $\mathrm{mAb}$, which generally binds to a different or repeating epitope on the antigen, forms a sandwich complex with the antigen and the capture antibody. The label yields a measurable, often optical signal. In a sandwich LFIA's working range, the $\mathbf{T}$ signal increases with an increase in the target antigen concentration; the naked eye can qualitatively read this. However, researchers have known since $1974^{4}$ that an excess antigen concentration leads to saturation of available binding sites on the bivalent capture and detector mAbs, preventing the formation of a sandwich complex in the $\mathbf{T}$ area, which in turn leads to a paradoxical loss of $\mathbf{T}$ signal intensity. $^{3,5-7}$ This disappearance of $\mathbf{T}$ is known as the hook effect. $^{8}$

In LFIA, in addition to the capture and detection mAbs, a secondary species-specific antibody, capable of binding the labeled detection $\mathrm{mAb}$, is immobilized as a control line (C). ${ }^{9}$ The $\mathbf{C}$ informs the user that the test is valid, yielding a signal regardless of the presence of an antigen. When analyzing LFIAs with a digital optical reader such as a smartphone, ${ }^{10}$ the everpresent $\mathbf{C}$ can be used to normalize the $\mathbf{T}$, to correct for experimental variables $(\mathrm{T} / \mathrm{C}) .^{11}$ The use of $\mathbf{T} / \mathrm{C}$ thus assumes that the $\mathbf{C}$ intensity is constant. However, it has been observed that an increasing antigen concentration also leads to a

Received: September 3, 2020

Accepted: November 2, 2020

Published: November 13, 2020 
decrease in the $\mathbf{C}$ intensity, while the $\mathbf{T}$ intensity still increases. $^{12-14}$ The loss of $\mathbf{C}$ compromises the reliability of the $\mathbf{T} / \mathrm{C}$ at high concentrations and yet remains to be fully understood. In the literature, various concentration-dependent effects are described under the hook-effect definition. See the Supporting Information, Table S1, for a review of the definitions given for the hook effect and the observed effects in the literature (1974-2020). Despite the qualifying characteristic of the hook-effect being a false negative result (i.e., the absence of $\mathbf{T}$ ), the definition is frequently also used to describe effects causing the loss of the $\mathbf{C}$.

There are numerous mitigation strategies to cope with highconcentration effects in sandwich immunoassays:

The most apparent method is testing the sample, both undiluted and diluted. ${ }^{15}$ If the diluted sample gives a stronger $T$ response than the undiluted sample, the undiluted result can be considered as "hooked". ${ }^{16}$ Dilutions allow adjustment of the dynamic working range of an LFIA but also require additional sample preparation, time, and material costs. ${ }^{17}$ Conversely, changing the physical layout of the assay can prevent highconcentration effects, ${ }^{2,18}$ essentially allowing for decoupled reagent delivery. ${ }^{19}$ Still, the separation of reagent flow is crucial to prevent premature mixing of the labeled antibody and analyte. $^{20}$ Alternatively, high-concentration effects can be minimized by optimizing reagents, for example, by supplementing the immunoassay with one or more additional target lines. ${ }^{6,21,22}$ Differentiation between artificially low ("hooked") and truly low concentration samples is possible by the realtime monitoring of $\mathbf{T}$ and $\mathbf{C}$ development ${ }^{14}$ and can allow for LFIA dynamic ranges to be expanded by orders of magnitude. $^{23}$

In this work, the aim was to first unravel the hook effect by comprehensively elucidating how extreme antigen concentrations influence the LFIA test line and control line development over time in three different allergen LFIAs. The identification and understanding of how high-antigen concentrations influence LFIA signal development is crucial for any developed sandwich LFIAs and will lead to ways to mitigate such effects, such as the simplified dynamic smartphone-based method presented here, ultimately leading to more reliable testing.

\section{EXPERIMENTAL SECTION}

Three allergen LFIAs were developed for detecting peanut (PA) and hazelnut (HA1 + HA2). Each assay has a different sandwich pair of mAbs for their capture $(T)$ and detector carbon nanoparticle-labeled-antibody (CNP-mAb), selected for their differences in sensitivity as observed in prior works. $^{24-26}$ All assays used goat anti-mouse (GAMaB) IgG in PBS ( $\mathrm{pH} 7.6 ; 1.2 \mathrm{mg} / \mathrm{mL}$; AffiniPure $\mathrm{F}\left(\mathrm{ab}^{\prime}\right)_{2}$ Fragment) at the $\mathbf{C}$ line (Jackson Immunoresearch Laboratories Inc., Sanbio, Uden; The Netherlands) and were developed on nitrocellulose membranes (140 CN; Unisart, Sartorius, Gottingen, Germany) overlaid with an absorbent pad (Whatman, GE Healthcare, Eindhoven, The Netherlands) and secured with a plastic backing ( $G$ and L, San Jose, CA, USA); see Supporting Information Protocol S1 and S2 for full details on CNP-mAb labeling and LFIA preparation details. For the surface plasmon resonance (SPR) biosensor assay, an amine coupling kit, $\mathrm{pH}$ scouting kit, HBS-EP buffer, and CM5 sensor chips were purchased from GE Healthcare (Uppsala, Sweden); see Supporting Information Protocol S3 for further details on the immobilization procedure.
Reference Material Preparation. Standardized certified reference materials and standard solutions for food allergens are not currently available, and therefore, the total protein extracts used here required in-house preparation. ${ }^{27}$ The procedure for the protein extraction for generating total peanut protein (TPP) and total hazelnut protein (THP) has been described previously. ${ }^{25,26}$ Fresh aliquots were defrosted on the day of the experiment, and the protein content was checked with the NanoDrop (ND 3300, Isogen Life Sciences, De Meern; The Netherlands) before use.

LFIA Readout. A qualitative assessment of LFIAs was made by reading the developed signal with the naked eye; quantitative readings were performed by smartphone detection. ${ }^{28}$ A custom 3D-printed smartphone holder was used to shield up to three LFIAs from ambient light during the optical measurements (See Figures 1, S1 and S2). A smartphone
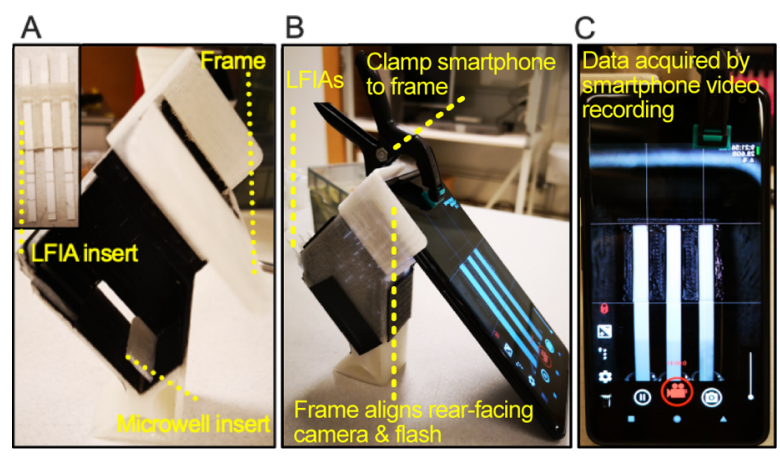

Figure 1. Photographs of the 3D-printed smartphone holder for recording LFIA signal development under controlled lighting: (A) open side view to show where LFIAs and microwells are inserted; (B) closed side view with the LFIAs inserted and smartphone attached; and $(\mathrm{C})$ head-on view of LFIA signal development on the phone screen.

(Google Pixel 2 XL, Google, Mountain View, CA; USA) was used to record images and videos of developing LFIAs. The smartphone was attached to the frame of the holder for support during the dynamic measurements (locked exposure, fixed focus, and controlled illumination) at 30 frames per second (fps) using OpenCamera (v1.47.3). Adapter (v2.1.6) converted the videos into images of $1 \mathrm{fps}$. Image ${ }^{29}$ was used to split the images into their RGB (red, green, and blue) color channels. Blue channel intensity values for the $\mathbf{T}$ and $\mathbf{C}$ were subtracted from a background reading; the resulting corrected blue channel pixel intensity (cBCPI) increased with increasing line intensity.

Influence of Antigen Concentration on LFIA Signal Development. All three LFIAs were tested in a concentration range spanning 5 orders of magnitude. The LFIAs were inserted into microwells containing $99 \mu \mathrm{L}$ of antigen in $\mathrm{RB}$ (0.0075-3500 ppm of TPP or THP) and $1 \mu \mathrm{L}$ of CNP-mAb. Here, the endpoint images of $\mathbf{T}$ and $\mathbf{C}$ signals were used for calculating T/C; LFIAs were left to develop for $40 \mathrm{~min}$ before the results were recorded using a smartphone.

Influence of Antigen Concentration on Signal Development in C-Only LFIAs. The LFIAs were tested alongside an LFIA with only a GAMaB C, at concentrations shown to diminish $\mathbf{C}$ intensity $(5,10,25$, and $50 \mathrm{ppm})(n=2)$. Additionally, the original LFIAs were run in a blank as a negative control. The LFIAs were developed in a microwell containing $99 \mu \mathrm{L}$ of antigen in RB (50 ppm TPP, 5 or $50 \mathrm{ppm}$ 
Peanut Assay

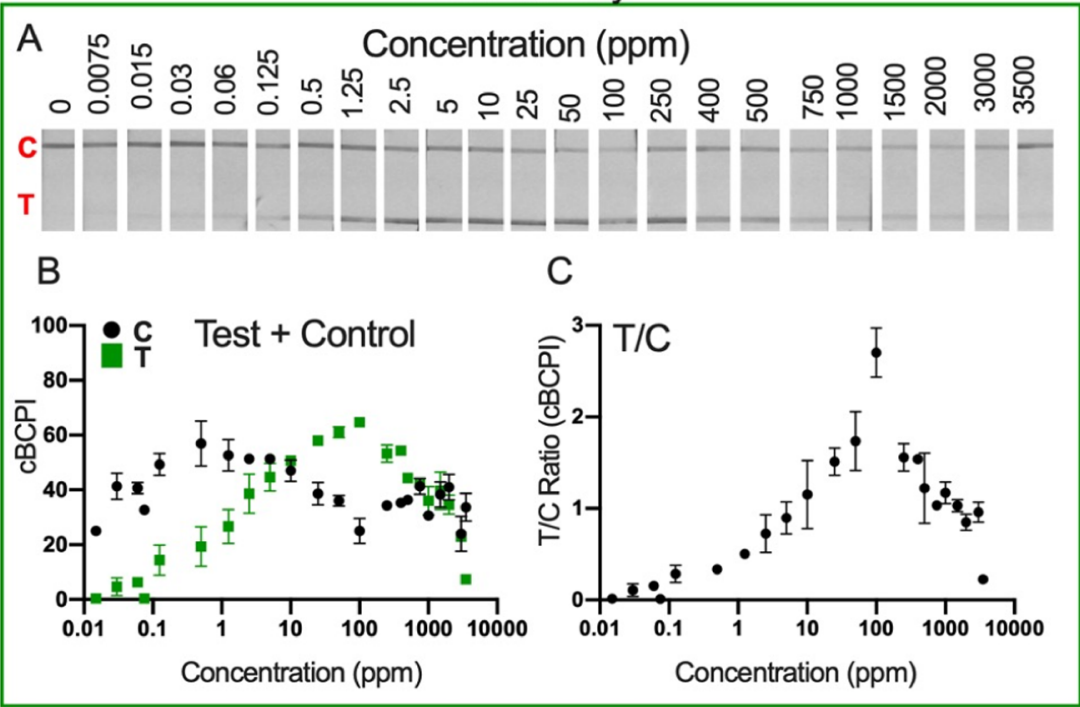

Hazelnut Assay 1

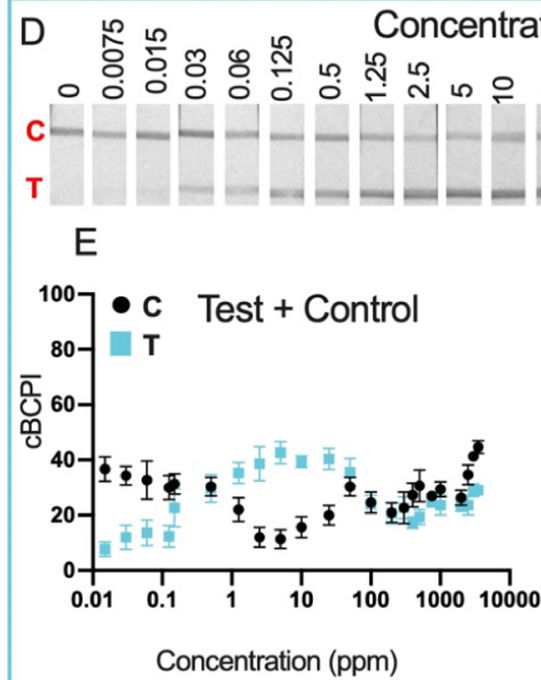

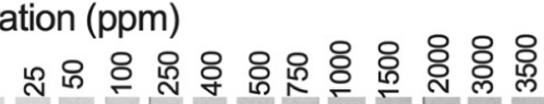

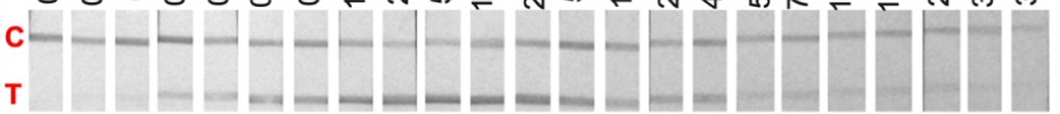

E

$\mathrm{F}$

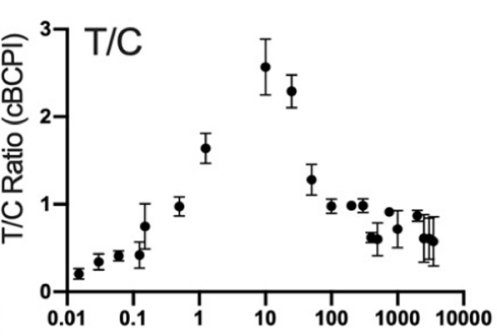

Concentration (ppm)

Hazelnut Assay 2

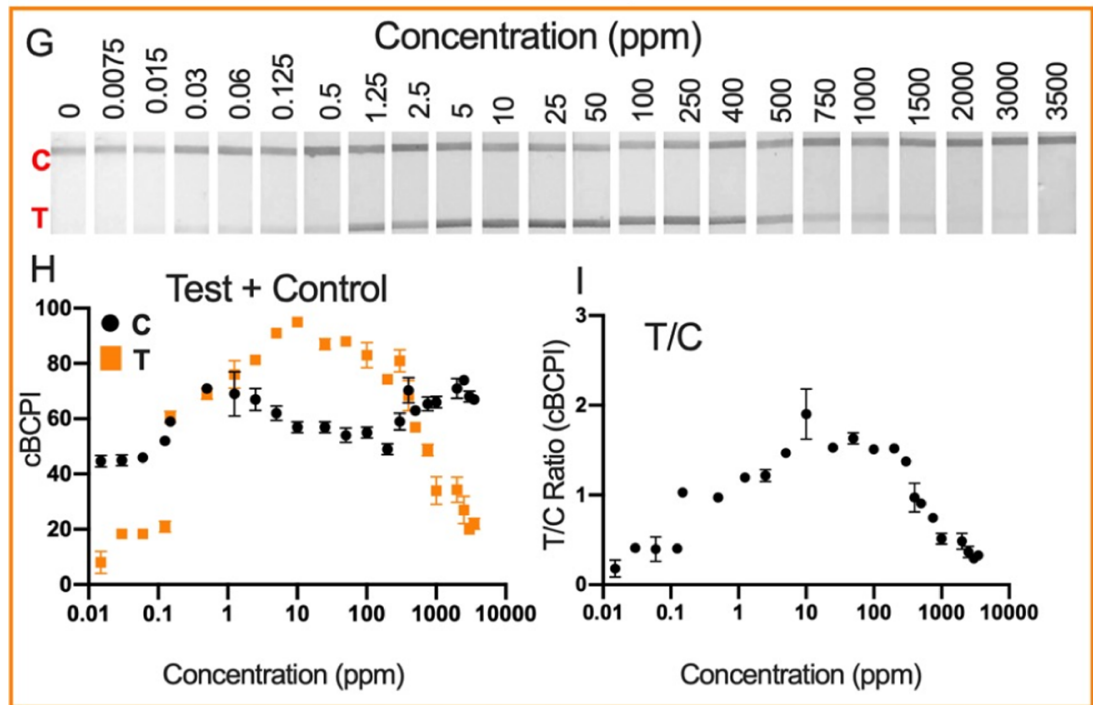

Figure 2. Extended calibration range of the three LFIAs, peanut assay [PA], hazelnut assay 1 [HA1], and hazelnut assay 2 [HA2] in increasing concentration of the analyte (TPP or THP) spiked into RB $(0.0075-3500 \mathrm{ppm})$. (A,D,G) Photographs after 40 min, (B,E,H) test and control signal expressed in $\mathrm{cBCPI}$, and $(\mathrm{C}, \mathrm{F}, \mathrm{I})$ test line divided by the control line $(\mathrm{T} / \mathrm{C}$ ratio). Error bars represent the standard deviation $(n=3)$. 
THP) and $1 \mu \mathrm{L}$ of CNP-mAb; the LFIAs were developed for $30 \mathrm{~min}$ in a $3 \mathrm{D}$-printed smartphone holder (Figure 1).

Sequential and Premixed Antigen-Binding Studied by SPR. Biosensor chip immobilization was performed per the manufacturer's instructions; see Supporting Information Protocol S3 for further details. Using SPR, the influence of increased analyte concentration on the binding characteristics of free CNP-mAb (and subsequently introduced analyte) or premixed immunocomplex (CNP-mAb-analyte) toward immobilized GAMaB was evaluated $(n=3)$. For sequential measurements, $10 \mu \mathrm{L}$ of CNP-mAb (diluted 1:99 in HBS-EP) was injected across the GAMaB surface (flow rate of $25 \mu \mathrm{L}$ / min). After binding, $10 \mu \mathrm{L}$ of analyte solution was injected. For premix experiments, $10 \mu \mathrm{L}$ of CNP-mAb and analyte solution was injected at $25 \mu \mathrm{L} / \mathrm{min}$. These experiments were performed at concentrations of $0.1-3000 \mathrm{ppm}$. For regenerating the surface, the flow was adjusted $(50 \mu \mathrm{L} / \mathrm{min})$ and $5 \mu \mathrm{L}$ of 25 $\mathrm{mM} \mathrm{NaOH}$ was injected, returning the signal to baseline.

Dynamic Monitoring of LFIA Signal Development. The LFIAs were placed in microwells containing $99 \mu \mathrm{L}$ of antigen in RB (0.0075-3500 ppm of TPP or THP) and $1 \mu \mathrm{L}$ of CNP-mAb, inserted into the smartphone holder, and were dynamically recorded for $30 \mathrm{~min}$. The $\mathrm{T} / \mathrm{C}$ was acquired at set time points $(5-30 \mathrm{~min})$ by selecting frames from the video. This was done for different concentrations [(PA; 0.015-3000 ppm), (HA1: 0.015-3000 ppm), and (HA2: 0.015-2000 $\mathrm{ppm})$ ]. Alternatively, the videos were imported into custom python scripts (Python 3.8) for automated data analysis. In an early video frame, regions of interest (ROI) were positioned over the $\mathbf{T}$ and $\mathbf{C}$, and as a background reading. Data evaluation consisted of averaging the blue pixel intensity in the ROIs across the entire video duration at 1 second data points. The generated responses were exported in a comma-separated value (.csv) format for easy importing into spreadsheet programs. A second complementary python script corrected the time response to assess LFIA signal development.

\section{RESULTS AND DISCUSSION}

Influence of Antigen Concentration on LFIA Signal Development. As most reported LFIAs are tested within a limited range and read after up to $20 \mathrm{~min}$, high concentration effects are not well documented, but it is known that excess antigen concentrations can influence the signal development time. Here, three different LFIAs were tested in a concentration range spanning 0.0075-3500 ppm (Figure 2) and developed over $40 \mathrm{~min}$ before their endpoint image was recorded using a smartphone.

In all three assays, the $\mathbf{T}$ follows the same pattern across the concentration range, as can be observed visually (Figure $2 \mathrm{~A}, \mathrm{D}, \mathrm{G}$ ) and numerically (Figure 2B,E,H). The $\mathbf{T}$ signal depends on the capture of antigen followed by binding of CNP-mAb, or on the binding of already complexed [CNP$\mathrm{mAb}$-analyte] to the immobilized antibody. At $0 \mathrm{ppm}$, there is no analyte present; therefore, no $\mathbf{T}$ signal develops. As the analyte concentration increases [up to $100 \mathrm{ppm}$ (PA), $10 \mathrm{ppm}$ (HA1), and $10 \mathrm{ppm}(\mathrm{HA} 2)]$, the $\mathbf{T}$ intensity increases by capturing more analyte and correspondingly more $\mathrm{CNP}-\mathrm{mAb}$ or by capturing larger, higher-order immunocomplexes. These complexes form when multivalent antigens, such as hazelnuts and peanuts, with numerous identical or distinct epitopes, ${ }^{30}$ bind several CNP-mAb particles, leading to the formation of an intense $\mathbf{T}$ at high concentrations. Beyond these concentrations, the $\mathbf{T}$ intensity instead starts decreasing, producing false negatives. This hook effect is unsurprising because, at extreme antigen-excess, $\mathbf{T}$ is rapidly saturated by an accumulation of unlabeled antigen, while the remaining mobile, excess antigen binds with CNP-mAb without being captured on $\mathbf{T}{ }^{2}$

Comparably, the $\mathbf{C}$ signal arises because of the binding of CNP-mAb. Therefore, in a blank sample $(0 \mathrm{ppm})$, a clear $\mathbf{C}$ is seen. At low concentrations, $\mathbf{C}$ captures free CNP-mAbs, yielding an intense signal, as observed visually (Figure $2 \mathrm{~A}, \mathrm{D}, \mathrm{G}$ ) and numerically (Figure 2B,E,H). In this range, $\mathbf{C}$ intensity increases slightly with increasing antigen concentration, possibly because of the binding of multiple CNP-mAbs to the same multivalent allergenic protein, resulting in increased signal intensity. ${ }^{31}$ At higher concentrations [roughly above 10 ppm (PA), $0.5 \mathrm{ppm}$ (HA1), and 1 ppm (HA2)], the C intensity decreases, while the $\mathrm{T}$ still becomes more intense. This is reflected in the endpoint T/C metric (Figure 2C,F,I). $\mathbf{T} / \mathbf{C}$ increases along with $\mathbf{T}$ and rises further even at concentrations causing $\mathbf{C}$ to decrease. This increase in $\mathbf{T} / \mathbf{C}$ widens the LFIA's linear dynamic working range even when $\mathbf{C}$ is affected by the concentration. Only after the hook-effect has occurred, leading to a decrease in $\mathbf{T}$, does the $\mathbf{T} / \mathbf{C}$ drastically drop. This trend is consistent across all three assays, despite them detecting distinct antigens and using different $\mathrm{mAb}$ sandwich pairs with diverse sensitivities and kinetics; ${ }^{24,25}$ as well as from the body of literature describing high concentration effects in LFIA (see Supporting Information Table $\mathrm{S1}^{2,12,14}$ ). A decrease in $\mathbf{C}$ must be caused by a reduction in CNP-mAb binding, which could potentially be due to preventing the arrival of CNP-mAbs at the $\mathbf{C}$ by the $\mathbf{T}$ (investigated in the $\mathrm{C}$-only section below) or decreasing the avidity of the CNP-mAbs to bind at those sites, which is assessed by the SPR experiments. ${ }^{32}$

Interestingly, as the concentration further increases [above $100 \mathrm{ppm}$ (PA) and $250 \mathrm{ppm}$ (HA1 and HA2)], the $\mathbf{C}$ intensity partially reappears. At extreme concentrations, multivalent proteins have a propensity to aggregate, potentially masking their epitopes. ${ }^{33}$ Moreover, these antigen concentrations probably hinder higher-order immunocomplex formation because there is insufficient CNP-mAb available for binding with the abundant antigen in larger complexes. ${ }^{12,34}$

Influence of Antigen Concentration on Signal Development in C-Only LFIAs. In the extended calibration range, specific concentrations caused the appearance of an intense $\mathbf{T}$ and a comparably diminished $\mathbf{C}$, affecting the $\mathbf{T} / \mathbf{C}$, as was observed in Figure 2C,F,I. Possibly, at these moderately high concentrations, the antigen, which is bound in the immunocomplex with CNP-mAb, binds mostly at the $\mathrm{T}$, thus limiting the amount of CNP-mAb that can reach the $\mathrm{C}$ and can interact with GAMaB.

Figure $3 \mathrm{~A}-\mathrm{C}$ shows the three assays tested at concentrations observed to affect $\mathrm{C}$ development; Figure $3 \mathrm{D}$ shows the cBCPIs $(n=2)$ of the $\mathbf{C}$ from the three variations of the assay, for all the three assays. Both in the presence and absence of a $\mathbf{T}$, the $\mathbf{C}$ never reaches the full intensity it would reach in a blank. Interestingly, the signal for the $\mathrm{C}$ in the regular LFIA [green (PA), blue (HA1), and orange (HA2)] and the C-only LFIA [checked green (PA), checked blue (HA1), and checked orange (HA2)] are of similar intensity. However, 3D shows that the intensity of $\mathbf{C}$ in the $\mathbf{C}+\mathbf{T}$ LFIAs is often less than in the C-only LFIAs, indicating that some binding of [CNP-mAbanalyte ] at $\mathbf{T}$ could have a minor contribution to the reduction of binding at $\mathbf{C}$. However, there is a more substantial $\mathbf{C}$ intensity difference between the blank and antigen solutions 


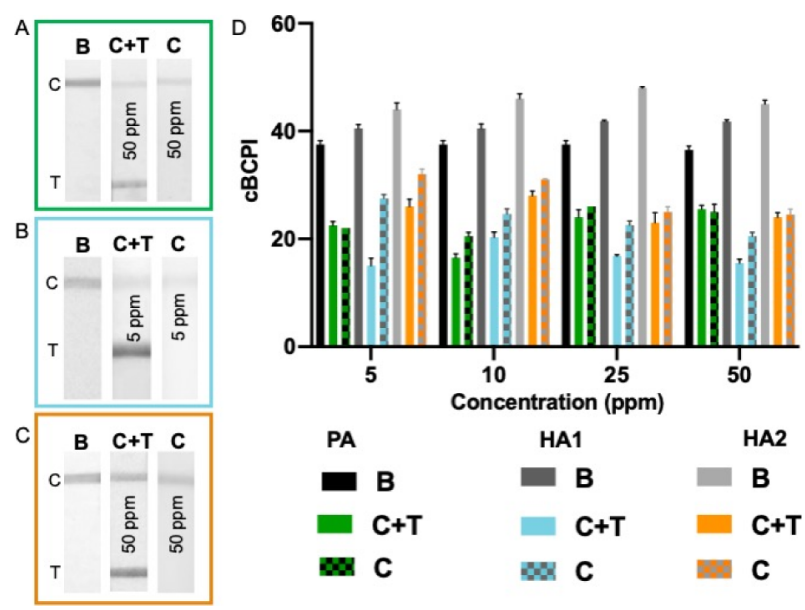

Figure 3. Control line-only LFIAs. Control signal development in LFIAs with a control line and a test line $(\mathbf{C}+\mathbf{T})$ and LFIAs with only a control line (C). (A) Peanut assay (PA) C $+\mathrm{T}$ signal development in a blank (B), C $+\mathbf{T}$ and $\mathbf{C}$ in $50 \mathrm{ppm}$ TPP. (B) Hazelnut assay 1 (HA1) $\mathbf{C}+\mathbf{T}$ and $\mathbf{C}$ signal development in $\mathbf{B}$ and in 5 ppm THP, (C) hazelnut assay 2 (HA2) $\mathbf{C}+\mathbf{T}$ and $\mathbf{C}$ signal development in $\mathbf{B}$ and in $50 \mathrm{ppm}$ THP, and (D) signal intensity in $\mathbf{B}, \mathbf{C}+\mathbf{T}$ and $\mathbf{C}$ across all three LFIAs as a cBCPI.

for each assay, at all tested concentrations. This emphasizes that a reduction of binding at $\mathbf{C}$ must be due to the increased antigen concentrations, causing the CNP-mAb to have a decreased avidity for $\mathbf{C}$.

Sequential and Premixed Antigen-Binding Studied by SPR. Here, using SPR, we set out to elucidate whether increased antigen concentration hinders the labeled [CNPmAb-analyte] immunocomplex' ability to bind with the $\mathrm{GAMaB}$ (premix) compared with whether increased antigen concentration affects the binding of CNP-mAb decoupled from the analyte. SPR typically is label-free, ${ }^{24}$ but antibody labeling can alter essential binding characteristics. ${ }^{35,36}$ In SPR, response units (RUs) are generated by the total amount of material captured at the surface, compared with LFIA, where the signal is made up solely from the binding of CNP-mAb to C. Moreover, it is also important to note that these assays take place on a very different time scale (i.e., LFIA; $40 \mathrm{~min}$ vs SPR; $40 \mathrm{~s})$.

The RU's reproducibly increased in all three assays following the injection of CNP-mAb, as can be seen in Figure 4A-C, where the black bar represents the free CNP-mAb binding with $\mathrm{GAMaB}$. Adjusting the concentration of the injected analyte in the second step again leads to a reproducible RU increase in all assays, as can be seen by the increasing colored bar [green (PA), blue (HA1), orange (HA2)]. Because these multivalent antigens bind to the captured CNP-mAb, thereby increasing the total mass of material bound to the chip surface, this increase in RU is unsurprising.

Contrastingly, when simultaneously injecting CNP-mAb + antigen (premix), the assays behaved differently [checked green (PA), checked blue (HA1), and checked orange (HA2)] (see Supporting Information Figure S3 for an example of sequential and premixed TPP sensorgrams). Crucially, the signal intensity in the premix approach is consistently lower versus the sequential approach at all tested antigen concentrations. In excess analyte concentrations, [CNP-mAbanalyte] quickly forms, depleting the free CNP-mAb, which would otherwise interact with $\mathbf{C}$ with higher avidity than the
A

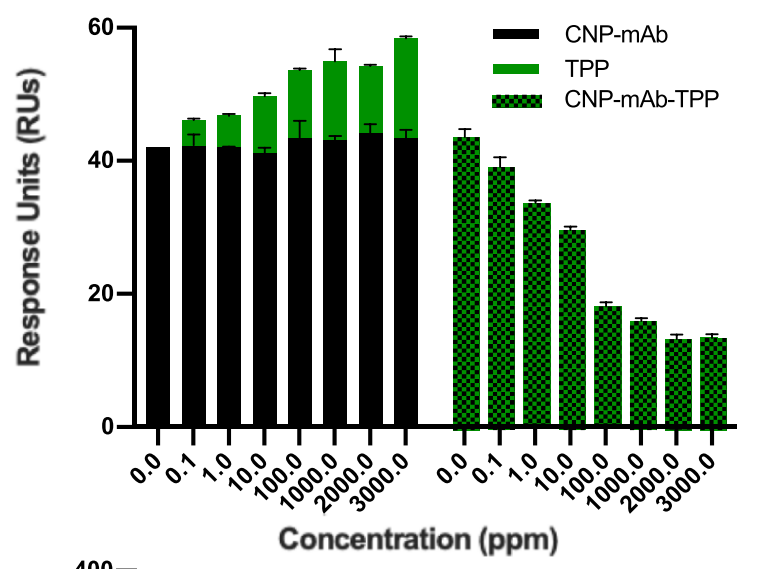

B

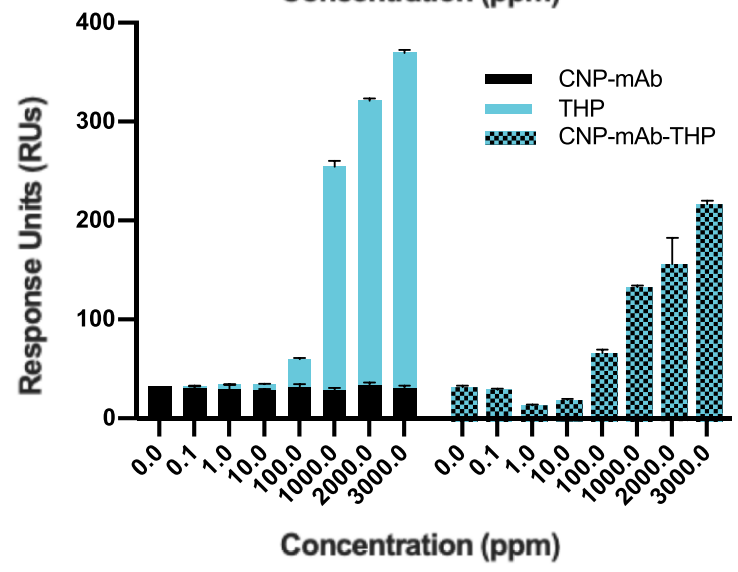

C

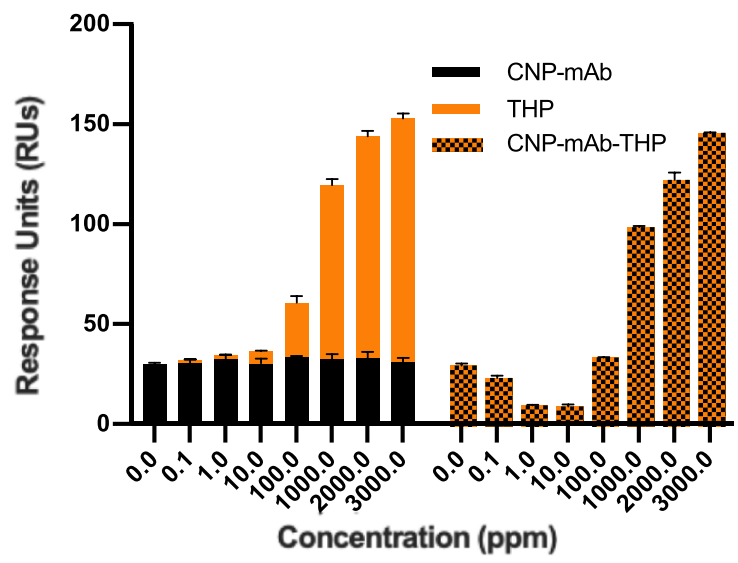

Figure 4. SPR responses showing binding to goat anti-mouse antibody of (A) total peanut protein [PA], (B) total hazelnut protein 1 [HA1], and (C) total hazelnut protein 2 [HA2] tested by sequentially injecting the carbon nanoparticle-labeled antibody (CNP-mAb; black) followed by antigen [green (PA), blue (HA1), and orange (HA2) ] compared against premixed CNP-mAb + antigen [checked green (PA), checked blue (HA1), and checked orange $(\mathrm{HA2}])](n=3)$. Standard deviation is expressed as error bars $(n=$ $3)$.

complex. ${ }^{2}$ This explains why high antigen concentrations cause a reduction in binding toward GAMaB in both LFIA and SPR. The $\mathbf{C}$ signal is consistently reduced at a lower concentration than the $\mathbf{T}$ signal in LFIA. As soon as an assay is in antigen excess, a prerequisite to enter the hook-range, higher-order immunocomplexes would already have formed in the solution. Formation of such complexes would deplete the amount of free CNP-mAb available for binding with $\mathrm{C}$. 


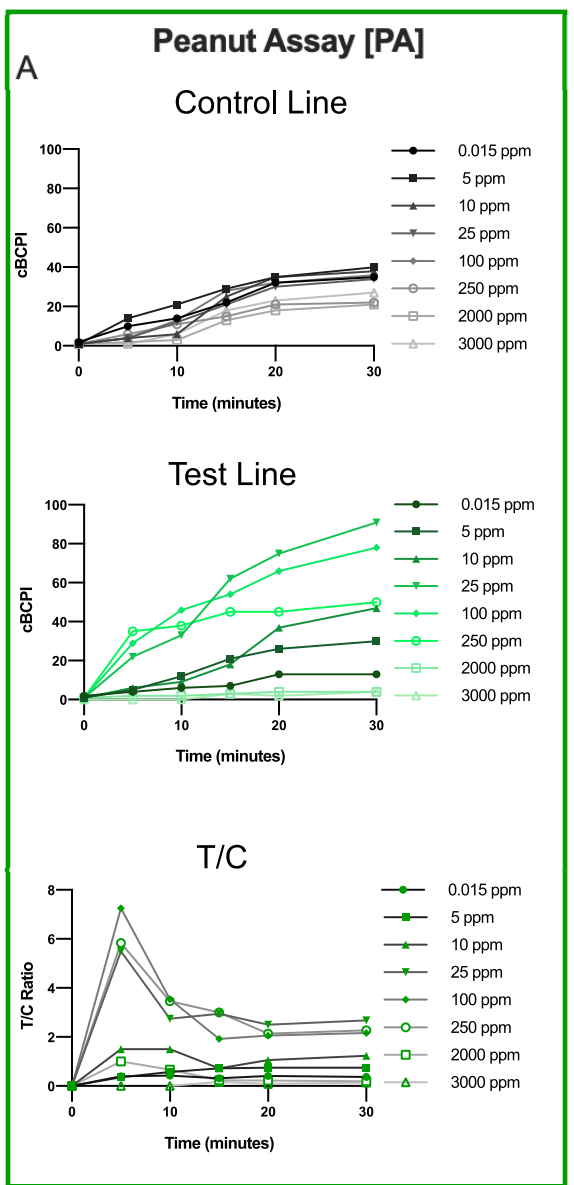

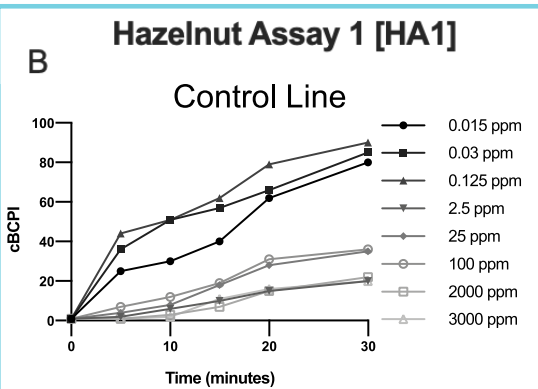

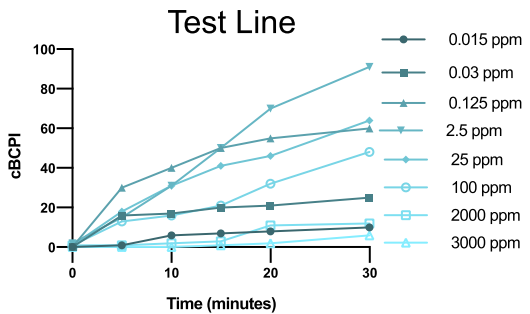

$\mathrm{T} / \mathrm{C}$

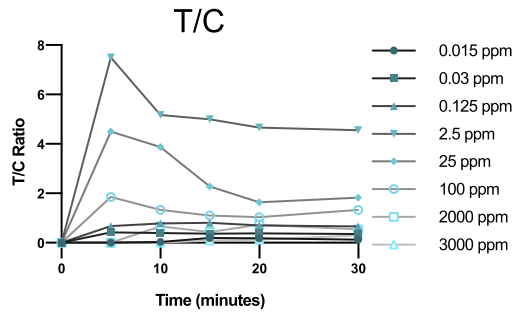

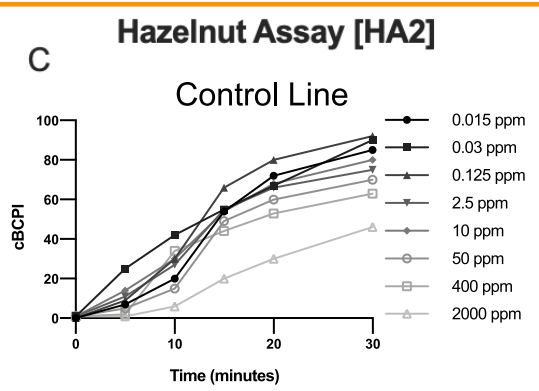

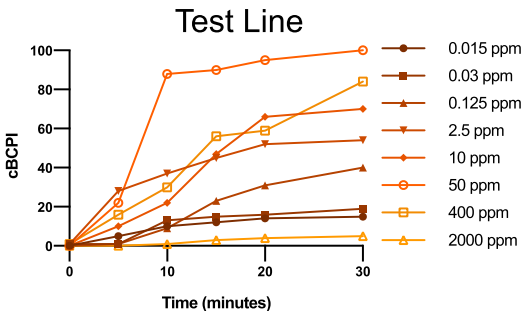

$\mathrm{T} / \mathrm{C}$

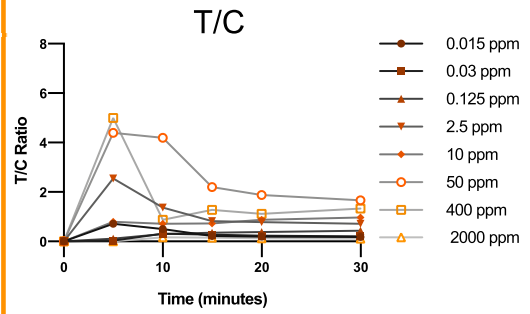

Figure 5. Dynamic smartphone monitoring of signal development for total peanut protein (PA), total hazelnut protein 1 (HA1), and total hazelnut protein 2 (HA2) LFIAs. Control line signal development (A) [PA], (B) [HA1], and (C) [HA2]; test line signal development (D) [PA], (E) [HA1], and (F) [HA2]; and T/C ratio development (G) [PA], (H) [HA1], and (I) [HA2] over 30 min at different concentrations [0.015-5 ppm (PA)] [0.015-0.125 ppm (HA1 and 2)].

Therefore, for the hook effect at $\mathbf{T}$ to occur, the concentration effect on the $\mathbf{C}$ must have already taken place.

The response in both [HA] assays is different from the [PA] assay, although this is not surprising, considering that all the assays use different antibodies with varied sensitivities and detect distinct analytes. In the $[\mathrm{PA}]$, the total binding decreases as antigen concentration increases, which is consistent with the formation of a higher number of immunocomplexes with reduced avidity to GAMaB. Previously, using SPR, Liang ${ }^{2}$ observed a concentration-dependent decrease in labeled immunocomplex binding toward $\mathbf{T}$, but did not study how concentration influences complex binding at C. Here, it is clear that there is a decrease in binding at GAMaB with increasing TPP concentrations, seemingly harmonious with a growing number of complexes. In both [PA] formats, a decrease in $\mathbf{C}$ signal is observed at high concentrations, but this stabilizes going up to $3000 \mathrm{ppm}$. In the LFIA, one additional higher concentration ( $3500 \mathrm{ppm})$ was also tested, and at this concentration, $\mathbf{C}$ partially recovered.

Contrastingly, in both [HA] LFIAs, we see a reappearance of $\mathrm{C}$ at much lower concentrations than with $[\mathrm{PA}]$, which is logical, considering that the $[\mathrm{HA}]$ LFIAs are more sensitive than the $[\mathrm{PA}]$. Likewise, both premixed HAs initially decrease in RU with increasing concentration $(<100 \mathrm{ppm})$, consistent with the formation of higher-order complexes with reduced avidity for $\mathbf{C}$; these concentrations are also observed to cause a reduction in $\mathbf{C}$ in the corresponding LFIAs (Figures 2 and 3). However, at higher concentrations, both HA assays instead increase in RU; these concentrations likely cause protein aggregation and have a relative scarcity of CNP-mAbs compared to the overabundant antigen, inhibiting the formation of higher-order complexes. ${ }^{37}$

Dynamic Monitoring of LFIA Signal Development. The SPR data suggest that the binding of immunocomplexes differs from the binding of free CNP-mAb, resulting in variations of the $\mathbf{C}$ intensity across broad concentration ranges. The usefulness of $\mathbf{T} / \mathbf{C}$ for normalizing sandwich LFIA results is impeded across this range when considering only the endpoint analysis. However, recent research suggests that additional information is available by monitoring the development of the $\mathbf{T} / \mathbf{C}$ over the entire assay duration. ${ }^{14}$ Figure 5 shows the signal development of the $\mathbf{C}$ (Figure $5 \mathrm{~A}-\mathrm{C}$ ), $\mathbf{T}$ (Figure $5 \mathrm{D}-\mathrm{F}$ ), and $\mathrm{T} / \mathrm{C}$ (Figure $5 \mathrm{G}-\mathrm{I}$ ) of the LFIAs at a range of TPP or THP concentrations [0.015-3000 ppm (PA)] [0.015-3000 ppm (HA1) and 0.015-2000 ppm (HA2)] (see Supporting Information Figure S4 for snapshots from the video recording of developing LFIAs).

Within the assay working range $[0.015-5 \mathrm{ppm}$ (PA)] [0.015-0.125 ppm (HA1 and 2)], the $\mathbf{C}$ always develops faster than or at the same time as the $\mathbf{T}$, resulting in a low, stable $\mathbf{T}$ / C over time. With increasing concentrations $[10-250 \mathrm{ppm}$ (PA)], [2.5-100 ppm (HA1)], and [2.5-400 ppm (HA2)], 
Table 1. Summary of Results of Concentration-Dependent Effects Studied in This Work

\begin{tabular}{|c|c|c|c|}
\hline experiment & purpose & result & conclusions \\
\hline \multirow[t]{4}{*}{$\begin{array}{l}\text { influence of concentration on } \\
\text { LFIA signal development }\end{array}$} & $\begin{array}{l}\text { investigate } \mathbf{T} \text { and } \mathbf{C} \text { and } \mathbf{T} / \mathbf{C} \text { development } \\
(0.0075-3500 \mathrm{ppm})\end{array}$ & C increases at low $[$ conc $]$ & hook effect is reproducible \\
\hline & & $\begin{array}{l}\mathbf{T} \text { increases at high }[\text { conc], at extreme } \\
{[\text { conc] } \mathbf{T} \text { is lost (hook effect) }}\end{array}$ & \\
\hline & & $\mathbf{C}$ decreases at a lower $[$ conc] than $\mathbf{T}$ & $\begin{array}{l}\text { C decreases because of reduced } \\
\text { CNP-mAb binding or due to } T \\
\text { depleting CNP-mAb }\end{array}$ \\
\hline & & C partially recovers at extreme [conc] & \\
\hline $\begin{array}{l}\text { influence of concentration on } \\
\text { signal development in C only } \\
\text { LFIAs }\end{array}$ & determine how $\mathbf{C}$ signal develops without a $\mathbf{T}$ & $\begin{array}{l}\text { high }[\text { conc] prevents } \mathbf{C} \text { from reaching } \\
\text { the same intensity as in a blank }\end{array}$ & high $[$ conc] negatively affects $\mathbf{C}$ \\
\hline \multirow[t]{3}{*}{$\begin{array}{l}\text { sequential and } \\
\text { premixed antigen-binding } \\
\text { studied by SPR }\end{array}$} & $\begin{array}{l}\text { determine the difference between premix and sequential } \\
\text { binding of CNP-mAb and antigen to GAMaB } \\
(0.1-3000 \mathrm{ppm})\end{array}$ & $\begin{array}{l}\text { sequential }[\mathrm{PA}],[\mathrm{HA} 1],[\mathrm{HA} 2]: \text { at high } \\
\quad[\text { conc] RUs increase }\end{array}$ & $\begin{array}{l}\text { free CNP-mAb has higher avidity to } \\
\text { GAMaB than [CNP-mAb-analyte] } \\
\text { complexes }\end{array}$ \\
\hline & & $\begin{array}{l}\text { premix }[\mathrm{PA}] \text { : at high }[\text { conc] RUs } \\
\text { decrease }\end{array}$ & \\
\hline & & $\begin{array}{l}\text { premix }[\mathrm{HA} 1]+[\mathrm{HA} 2]: \text { at low }[\text { conc }] \\
\text { RUs decrease and at high }[\text { conc }] \text { RUs } \\
\text { increase }\end{array}$ & \\
\hline \multirow[t]{3}{*}{$\begin{array}{l}\text { dynamic monitoring of LFIA } \\
\text { signal development }\end{array}$} & $\begin{array}{l}\text { investigate } \mathbf{T}, \mathbf{C} \text { and } \mathbf{T} / \mathbf{C} \text { development over time at } \\
\text { varying concentrations }\end{array}$ & $\begin{array}{l}\text { dynamic working range: } \mathbf{T} \text { and } \mathbf{C} \text { at a } \\
\text { similar time }\end{array}$ & $\begin{array}{l}\text { dynamic monitoring of } \mathbf{T} \text { and } \mathbf{C} \text { can } \\
\text { distinguish high concentration ef- } \\
\text { fect }\end{array}$ \\
\hline & & $\begin{array}{l}\text { high }[\text { conc]: } \mathbf{T} \text { increases faster than } \mathbf{C} \text {; } \\
\mathbf{T} / \mathbf{C} \text { increases and then decreases }\end{array}$ & \\
\hline & & $\begin{array}{l}\text { extreme }[\text { conc]: no signal for approx. } \\
10 \mathrm{~min} \text {, then } \mathrm{C} \text { increases }\end{array}$ & \\
\hline
\end{tabular}

the speed of binding shifts, with the $\mathbf{T}$ developing more quickly than the $\mathbf{C}$. The $\mathbf{T} / \mathbf{C}$ time development reflects this with a sharp initial increase ( $5 \mathrm{~min})$, when mostly $\mathbf{T}$ is present, followed by a steady decline as the $\mathbf{C}$ belatedly develops and the signal balances out. Outside of the dynamic working range, when the $\mathbf{T}$ or $\mathbf{C}$ development is influenced by antigen concentration, the final $\mathbf{T} / \mathbf{C}$ becomes an unreliable metric. Recently, Rey ${ }^{14}$ presented a method for monitoring LFIA signal development, using a different label (i.e., gold nanoparticles), detecting another analyte (C-reactive protein), and similarly observed an initial rise, followed by a decrease in $\mathbf{T} / \mathrm{C}$ over the assay duration at concentrations where the $\mathbf{T}$ develops before the $\mathbf{C}$. Interestingly, here we observed this trend across all three LFIAs, albeit at different concentrations. Unfortunately, Rey's ${ }^{14}$ study primarily looked at T/C's in a limited antigen range $(120-255 \mu \mathrm{g} / \mathrm{mL})$, and as such only tested concentrations that caused a delayed and diminished development of $\mathbf{C}$ rather than the inhibition of $\mathbf{T}$ development. A recent study ${ }^{23}$ consolidated Rey's results by testing a different antigen (hCG) and modeling and observing $\mathbf{T} / \mathbf{C}$ development at different antigen concentrations $(0.5-500 \mathrm{IU} / \mathrm{mL})$ over 10 min. They found that at low hCG concentrations, C initially develops faster than $\mathbf{T}$, with $\mathbf{T} / \mathbf{C}$ increasing after $\mathbf{C}$ is saturated and $\mathbf{T}$ keeps developing over $10 \mathrm{~min}$. Conversely at high concentrations $\mathbf{T}$ develops rapidly with $\mathbf{C}$ developing slowly, causing $\mathbf{T} / \mathbf{C}$ to intially rise and afterwards to steadily decrease over the assay duration as $\mathbf{T}$ is saturated and $\mathbf{C}$ still increases. which is in line with our own findings.

Additionally, we found that a delayed and weak $\mathbf{C}$ development at higher concentrations also occurs when testing LFIAs without a T [50 ppm (PA), $25 \mathrm{ppm}$ (HA1), and 50 ppm (HA2)] (Supporting Information Figure S5), where the C signal only starts developing after $10 \mathrm{~min}$ and then only with a diminished intensity. As concluded from the SPR study, reduction in $\mathbf{C}$ intensity is mainly caused by the formation of (higher-order) immunocomplexes, which have reduced avidity for $\mathbf{C}$. While any $\mathbf{T}$ signal is weak, a diminished and delayed $\mathbf{C}$ eventually starts to develop after $10 \mathrm{~min}$, giving a final low $\mathrm{T} /$ C.

Critically, at these concentrations, the resulting low final $\mathbf{T}$ / C overlaps with the $\mathbf{T} / \mathbf{C}$ at much lower concentrations $[0.015$ ppm (PA), 0.015 and $0.03 \mathrm{ppm}$ (HA1) and (HA2)]; misinterpretation of this could lead to reporting of a false negative, as can be seen in Supporting Information Figure S6, where the $\mathbf{T}$ and $\mathbf{C}$ signal development and resulting $\mathbf{T} / \mathbf{C}$ are compared for HAl at $0.015 \mathrm{ppm}$ and $2000 \mathrm{ppm}$. While the final $\mathbf{T} / \mathrm{Cs}$ at these concentrations are similar, a false negative can be avoided by monitoring when the $\mathbf{T}$ and $\mathbf{C}$ develop; at hook-effect concentrations, no signal develops on either line for the first $10 \mathrm{~min}$, causing a static $\mathrm{T} / \mathrm{C}$ during this time. Comparatively, at low concentrations in the dynamic working range, the $\mathbf{C}$ signal develops rapidly and with a high intensity. By using dynamic video acquisition, real and artificially low concentration measurements can be differentiated, not only based on signal intensity but additionally based on whether the $\mathbf{C}$ or $\mathbf{T}$ develops first (Figure 5). Further, it is possible to automatically generate these $\mathbf{T}$ and $\mathbf{C}$ development profiles directly from the smartphone video (see Supporting Information Figure S7 for automatic profiles for PA compared with manual time-development graphs and Figure S8 for a blank assay), using the python script. In addition to dynamic intensity measurements, this allows for BCPI correction and autobackground subtraction. Considering that the results generated by the software correlate with the manually plotted time responses, novice users can simply use the automated results rather than carrying out the image analysis, corrections, and background subtraction themselves.

\section{CONCLUSIONS}

The experiments, results, and conclusions drawn have been summarized in Table 1 ; a schematic depiction of the complementary experiments can be found in Supporting Information Figure S9. To unravel the hook effect, we devised an inexpensive, dynamic, smartphone-based method for 
directly identifying the concentration-dependent effects across three different sandwich LFIAs. We comprehensively elucidated how both the antigen concentration and time influence signal development and allow us to differentiate between two distinct concentration effects: (1) the reduced development of a $\mathbf{C}$ in the presence of a rapidly and intensely developing $\mathbf{T}$, which occurs within the first few minutes and (2) the decrease in signal development on either line for $10 \mathrm{~min}$, followed by the development of a $\mathbf{C}$ which increases in intensity for the remaining assay duration. These trends were consistent across all three assays.

Indeed, we discovered for the hook effect to occur on $\mathbf{T}$, the concentration effect at $\mathbf{C}$ must have already happened. Based on our findings, we propose that a more appropriate definition for the moderate-high concentrations, which lead to the loss of the $\mathbf{C}$ in LFIA, would be the situation of free secondary antibody depletion. In free secondary antibody depletion, higher-order [CNP-mAb-analyte] immunocomplexes form, hindering the signal development at $\mathbf{C}$ by reducing the $\mathrm{CNP}-\mathrm{mAb}$ avidity for binding. As the concentration of analyte increases further, the assay starts to enter the hook-effect range. At these extreme concentrations, the unlabeled analyte rapidly saturates $\mathbf{T}$. We have established that while the endpoint $\mathbf{T} / \mathbf{C}$ is an appropriate metric within the dynamic working range, outside of this range when the test or control line is falsely diminished, the final T/ $\mathrm{C}$ is influenced. While a prediction algorithm is outside of the scope of this paper, we appreciate that this would be a useful advancement. Here, the automatically generated qualitative binding profiles provide a simplified way for novice users to monitor concentration effects without performing any image or data analysis themselves.

Further, the current system can simultaneously analyze three LFIAs, making it feasible to include an in-built quality control LFIA; such a control would be highly relevant where nonexperts, such as allergic consumers, perform LFIAs. Ultimately, the use of dynamic readout provides an inexpensive and direct mechanism for identifying the high-concentration effects in LFIA. The digital analysis of dynamic data allows clear differentiation between the highly concentrated samples and low concentration results. We foresee that this method should have broad applicability for distinguishing false-negative results in sandwich LFIAs.

\section{ASSOCIATED CONTENT}

\section{S1 Supporting Information}

The Supporting Information is available free of charge at https://pubs.acs.org/doi/10.1021/acs.analchem.0c03740.

Overview of hook-effect definitions; protocols for CNP$\mathrm{mAb}$ labeling, LFIA preparation and SPR procedures; 3D-printed attachments, SPR sensorgrams, photos of developing LFIAs, automatically generated kinetic profiles, and a schematic overview of paper (PDF)

\section{AUTHOR INFORMATION}

\section{Corresponding Author}

Gert IJ. Salentijn - Wageningen Food Safety Research (WFSR), Wageningen University \& Research, Wageningen 6700 AE, The Netherlands; Laboratory of Organic Chemistry, Wageningen University, Wageningen $6708 \mathrm{WE}$,

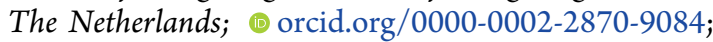
Email: gert.salentijn@wur.nl

\section{Authors}

Georgina M. S. Ross - Wageningen Food Safety Research (WFSR), Wageningen University \& Research, Wageningen 6700 AE, The Netherlands; 10 orcid.org/0000-0002-21452386

Daniel Filippini - Optical Devices Laboratory, Division of Sensor and Actuator Systems, IFM冈Linköping University, Linköping S58183, Sweden

Michel W. F. Nielen - Wageningen Food Safety Research (WFSR), Wageningen University \& Research, Wageningen 6700 AE, The Netherlands; Laboratory of Organic Chemistry, Wageningen University, Wageningen 6708 WE, The Netherlands; 10 orcid.org/0000-0003-4634-0249

Complete contact information is available at:

https://pubs.acs.org/10.1021/acs.analchem.0c03740

\section{Author Contributions}

G.M.S.R. conceptualization; methodology; validation; formal analysis; investigation; data analysis; writing original draft; writing-review and editing; project administration D.F. software; review and editing; M.W.F.N. resources; review and editing; supervision; funding acquisition; project administration G.IJ.S. conceptualization; methodology; writingreview and editing; supervision; project administration.

\section{Funding}

This project has received funding from the European Union's Horizon 2020 research and innovation program under the Marie Sklodowska-Curie grant agreement no. 720325.

\section{Notes}

The authors declare no competing financial interest.

\section{REFERENCES}

(1) Ross, G. M. S.; Bremer, M. G. E. G.; Nielen, M. W. F. Anal. Bioanal. Chem. 2018, 410, 5353-5371.

(2) Liang, T.; Robinson, R.; Houghtaling, J.; Fridley, G.; Ramsey, S. A.; Fu, E. Anal. Chem. 2016, 88, 2311-2320.

(3) Hoofnagle, A. N.; Wener, M. H. J. Immunol. Methods 2009, 347, $3-11$.

(4) Miles, L. E. M.; Lipschitz, D. A.; Bieber, C. P.; Cook, J. D. Anal. Biochem. 1974, 61, 209-224.

(5) Petakov, M. S.; Damjanović, S. S.; Nikolić-Durović, M. M.; Dragojlović, Z. L.; Obradović, S.; Gligorović, M. S.; Simić, M. Ž.; Popović, V. P. J. Endocrinol. Invest. 1998, 21, 184-188.

(6) Hu, J.; Choi, J. R.; Wang, S.; Gong, Y.; Feng, S.; PingguanMurphy, B.; Lu, T. J.; Xu, F. Sens. Actuators, B 2017, 243, 484-488. (7) Ki, H.; Oh, J.; Han, G.-R.; Kim, M.-G. Lab Chip 2020, 20, 844851.

(8) O'Farrell, B. Evolution in Lateral Flow-Based Immunoassay Systems. In Lateral Flow Immunoassay; Wong, R., Tse, H., Eds.; Humana Press: Totowa, NJ, 2009; pp 1-33.

(9) Kulabhusan, P. K.; Rajwade, J. M.; Sugumar, V.; Taju, G.; Sahul Hameed, A. S.; Paknikar, K. M. PLoS One 2017, 12, No. e0169012.

(10) Nelis, J. L. D.; Tsagkaris, A. S.; Dillon, M. J.; Hajslova, J.; Elliott, C. T. TrAC, Trends Anal. Chem. 2020, 129, 115934.

(11) Foysal, K. H.; Seo, S. E.; Kim, M. J.; Kwon, O. S.; Chong, J. W. Sensors 2019, 19, 4812.

(12) Posthuma-Trumpie, G. A.; Wichers, J. H.; Koets, M.; Berendsen, L. B. J. M.; van Amerongen, A. Anal. Bioanal. Chem. 2012, 402, 593-600.

(13) Pilavaki, E.; Demosthenous, A. Sensors 2017, 17, 2673.

(14) Rey, E. G.; O’Dell, D.; Mehta, S.; Erickson, D. Anal. Chem. 2017, 89, 5095-5100.

(15) Ruff, L. E.; Sapre, A. A.; Plaut, J. S.; De Maere, E.; Mortier, C.; Nguyen, V.; Separa, K.; Vandenbogaerde, S.; Vandewalle, L.; Esener, S. C.; Messmer, B. T. Nucleic Acids Res. 2016, 44, No. e96. 
(16) Anfossi, L.; Di Nardo, F.; Cavalera, S.; Giovannoli, C.; Baggiani, C. Biosensors 2018, 9, 2.

(17) Oh, J.; Joung, H.-A.; Han, H. S.; Kim, J. K.; Kim, M.-G. Theranostics 2018, 8, 3189-3197.

(18) Kim, T. H.; Hahn, Y. K.; Kim, M. S. Micromachines 2020, 11, 269.

(19) Tsai, T.-T.; Huang, T.-H.; Chen, C.-A.; Ho, N. Y.-J.; Chou, Y.J.; Chen, C.-F. Sci. Rep. 2018, 8, 17319.

(20) Hemmig, E.; Temiz, Y.; Gökçe, O.; Lovchik, R. D.; Delamarche, E. Anal. Chem. 2020, 92, 940-946.

(21) Shin, S.; Choi, M.; Shim, J.; Park, S. Sens. Actuators, B 2020, 304, 127408.

(22) Oh, Y. K.; Joung, H.-A.; Han, H. S.; Suk, H.-J.; Kim, M.-G. Biosens. Bioelectron. 2014, 61, 285-289.

(23) Sathishkumar, N.; Toley, B. J. Sens. Actuators, B 2020, 324, 128756.

(24) Bremer, M. G. E. G.; Smits, N. G. E.; Haasnoot, W. Anal. Bioanal. Chem. 2009, 395, 119-126.

(25) Ross, G. M. S.; Bremer, M.; Wichers, J. H.; van Amerongen, A.; Nielen, M. W. F. Biosensors 2018, 8, 130.

(26) Ross, G. M. S.; Salentijn, G. IJ.; Nielen, M. W. F. Biosensors 2019, 9, 143.

(27) Marsh, J. T.; Jayasena, S.; Gaskin, F.; Baumert, J. L.; Johnson, P. Food Chem. 2020, 313, 126019.

(28) Eriksson, E.; Lysell, J.; Larsson, H.; Cheung, K. Y.; Filippini, D.; Mak, W. C. Research 2019, 2019, 8079561.

(29) Schneider, C. A.; Rasband, W. S.; Eliceiri, K. W. Nat. Methods 2012, 9, 671-675.

(30) Taylor, S. L.; Nordlee, J. A.; Niemann, L. M.; Lambrecht, D. M. Anal. Bioanal. Chem. 2009, 395, 83-92.

(31) Qian, S.; Bau, H. H. Anal. Biochem. 2003, 322, 89-98.

(32) Ramos-Vara, J. A.; Miller, M. A. Vet. Pathol. 2014, 51, $42-87$.

(33) Shire, S. J.; Shahrokh, Z.; Liu, J. J. Pharm. Sci. 2004, 93, 13901402 .

(34) Koets, M.; Renström, A.; Zahradnik, E.; Bogdanovic, J.; Wouters, I. M.; van Amerongen, A. Journal of Environmental Monitoring 2011, 13, 3475-3480.

(35) Duo, J.; Bruno, J.; Kozhich, A.; David-brown, D.; Luo, L.; Kwok, S.; Santockyte, R.; Haulenbeek, J.; Liu, R.; Hamuro, L.; Peterson, J. E.; Piccoli, S.; DeSilva, B.; Pillutla, R.; Zhang, Y. J. Bioanalysis 2018, 10, 559-576.

(36) Duo, J.; Bruno, J.; Piccoli, S.; DeSilva, B.; Zhang, Y. J. Bioanalysis 2017, 9, 193-207.

(37) Bray, D.; Lay, S. Proc. Natl. Acad. Sci. U.S.A. 1997, 94, 1349313498 . 